\title{
Improving Performance of a Noise Reduction Algorithm by Switching the Analysis Filter Bank
}

\author{
Hamid Sepehr ${ }^{1,2}$, Amir Y. Nooralahiyan ${ }^{1}$, and Paul V. Brennan ${ }^{2}$ \\ ${ }^{1}$ ElaraTek LTD \\ ${ }^{2}$ University College London \\ h.sepehr@ucl.ac.uk
}

\begin{abstract}
A new approach for preservation of transient parts of speech in a noise reduction system is proposed in this paper. Transient components of speech such as vowel onset and beginning of some consonants such as stop sounds are important parts for intelligibility of speech. These components are usually attenuated by noise reduction algorithms due to the low temporal resolution of block-based noise reduction techniques. A method is proposed to detect the transient component of speech, followed by dynamic switching of the analysis filter bank at the front end of the noise reduction system to provide higher resolution in the time domain. The optimal spectral gain values are transformed into the time domain to form a linear filter in order to achieve noise reduction and only group delay equalisation is performed to avoid discontinuity. Our objective evaluation shows that the proposed method provides superior performance compared to noise reduction with fixed time/frequency resolution analysis filter banks.
\end{abstract}

\section{Introduction}

Ambient noise is the most common factor in degrading the quality and intelligibility of speech signals in many telecommunication networks, particularly when wireless communication devices, such as mobile or wireless headsets are used in high noise environments. Additionally, CODECs in many digital devices further reduce the quality and intelligibility of speech. The accumulative effect of CODECs and ambient noise can significantly reduce the quality of conversation in telecommunication networks, causing strain and listening fatigue to a user.

There are various known methods for performing noise estimation followed by noise cancellation on audio voice signals. One of the most practically used noise estimation approaches is based on minimum statistics [1] and its modified variants [2][3][4]. These methods are based on tracking the minimum value of energy in different sub-bands where the smoothed value of the minimum signal is considered as the estimated noise level. Other methods based on using a Voice Activity Detector (VAD) are also used [4] in which the estimated noise value is updated during speech pauses.

After the noise value is estimated, the majority of noise reduction part of the algorithm is performed in the spectral domain. The noise reduction algorithm transforms frame-by-frame of the audio data to the frequency domain and subsequently 
attenuates the estimated noise level, which usually results in some speech distortion. Two of the most practical (low complexity and ease of implementation) methods used for noise reduction are based on adaptive sub-band Wiener filtering and spectral subtraction performed independently in the frequency bins [4][5]. Equations (1) and (2) briefly describe the foundation of the spectral subtraction algorithm.

The noisy signal $y(m)$ is a sum of the desired signal $x(m)$ and the noise $n(m)$ :

$$
\begin{gathered}
y(m)=x(m)+n(m) \\
Y(\omega)=X(\omega)+N(\omega) \Rightarrow \tilde{X}(\omega)=Y(\omega)-\tilde{N}(\omega)
\end{gathered}
$$

Where $Y(\omega), X(\omega)$ and $N(\omega)$ are Fourier Transforms of $y(m), x(m)$ and $n(m)$, respectively. $N(\omega)$ and $X(\omega)$ respectively represent the estimated noise level and estimated clean speech signal.

The Short Time Fourier Transform (STFT) and Wavelet Transform are typical filter banks which are widely used in conjunction with different noise reduction algorithms such as Wiener filtering [6] and spectral subtraction [5] indicated above. One of the drawbacks of these methods is due to the fixed frame length of the audio data which results in fixed temporal/frequency resolutions of the selected transform method.

Commonly, the audio frame length is selected of sufficient length to obtain a good frequency resolution in order to achieve a good degree of noise reduction and to limit excessive variations in the noise reduction algorithm. This process is reasonable and can work well, however it does not take into account fast moving non-stationary elements of speech. This drawback results in non-optimal noise reduction performance in non-stationary transient parts of speech, creating pre-echo or undesirable attenuation of the wanted transient portion of the speech signal. Examples of such parts include plosive bursts, formant transitions and vowel onsets of speech, which are major contributors in intelligibility of speech signal.

The main area of study in this paper is to achieve a reasonable solution based on trading-off the frequency resolution when the transient components of speech signal are detected. The secondary aspect of this study is the time-domain implementation of the proposed solution and a review of the added advantages that this approach encompasses.

The above mentioned trade-off is addressed in a recent publication on noise reduction and speech enhancement. In [7], an adaptive buffer size is proposed to estimate and attenuate the noise. The adaptive segmentation algorithm defines the length of effective audio buffer size depending on the non-stationary nature of signal. In [8], time domain high temporal resolution audio data is used followed by an extrapolation technique to further improve the frequency resolution of the audio data, however this algorithm is computationally intensive and extrapolation doesn't necessarily guarantee improving the frequency resolution. Another approach is taken in [9] to mix the coefficients of two different analysis filter banks depending on transient detection of the signal, however, details of filter banks and complete reconstruction of the method is not addressed. The approach proposed in this study is somewhat similar to [10] in which a pair of adaptive analysis/synthesis filter banks 
with high/low temporal resolutions are switched (swapped) depending on the transient detection in the audio speech signal. The main difference in the approach presented in this study is that the system works in the time domain and the selected filter banks do not need to satisfy the full reconstruction restrictions during transient switching period.

In an interesting approach[11], similarity of visual and aural systems in human subjects and their sensitivity to the edge contour in image and acoustic edge (speech transient signal) in audio signal is exploited and an adaptive structure method (previously used for image enhancement [12]) is developed to enhance the speech signal.

In this paper, we propose a technique to switch between two different analysis filter banks depending on the transient detection in the signal, for which the process of noise reduction is carried out in the time domain. Objective measures and informal subjective testing demonstrate improvement in quality and intelligibility of the processed signal for a variety of noise test signals. The proposed algorithm has low complexity and requires a small memory footprint. It also introduces very low processing delay $(<8 \mathrm{msec})$ that makes it an attractive solution for many applications that are sensitive to processing delay in receive and/or transmit channels. Such applications include wireless handsets and network infrastructure, particularly enduser headset/handset and telephony devices with built-in side-tones.

In the next section the fundamentals of the suggested idea are outlined; section 3 describes the proposed algorithm for detection of transient parts of speech signal; in section 4 the modified structure of the proposed noise reduction is further explained; and finally the results and conclusion of the suggested method are presented in section 5 and 6.

\section{Fundamental Principals of the Suggested Method}

The fundamentals of the proposed algorithm lie in two concepts: (i) Detection of transient in speech signals in order to detect events such as vowel onset and plosive bursts. - this information is used to change the analysis filter bank to increase the temporal resolution of time-frequency representation of the signal; (ii) The other important aspect of the proposed algorithm is to perform the noise reduction method in the time domain as opposed to the traditional frequency domain.

The latter has been investigated by Gustafsson [13][14] where the main idea is to compute the optimal spectral gains based on priori SNR in different frequency bins. The frequency domain weights $w_{j}$ (j represents different sub band) are transformed to the time domain as a linear filter that is convolved with the time domain audio signal. As the frequency domain optimal weights are real value vectors, the time domain representation of these weights is a non-causal filter. This means random phase can be added to the spectral domain gains to achieve a causal real valued filter. A Hilbert-based algorithm is used in [13] to obtain the minimum phase filter. However, in this study, a linear phase approach is used for ease of implementation and in order to have a controlled group delay on the time domain filter, as shown in Equation 4. Equation 3, shows the optimal spectral domain gains for estimation of the 
desired signal. In Equation 4, $N$ is the length of the filter and hence the length of the audio buffer and $k$ represents different elements of the filter vector.

$$
\begin{gathered}
\tilde{X}(\omega)=Y(\omega) \times W(\omega)==>\tilde{x}(m)=y(m) * w(m) \\
{\left[w_{0}(m), w_{1}(m), \ldots, w_{N-1}(m)\right]=i f f t\left(\left[W_{0}(\omega), W_{1}(\omega), \ldots, W_{N-1}(\omega)\right] \times \exp (-\operatorname{sqrt}(-1) * 2 * p i * k / N)\right)}
\end{gathered}
$$

The proposed method for detection of transient elements of the audio signal is presented in the next section.

\section{Detection of Boundary of Speech Utterance}

In order to increase the temporal resolution of the proposed noise reduction, it is required to detect the points of interest and hence stationary boundaries of the audio signal. Transient detection has many applications in audio signal processing from efficient audio coding, audio signal segmentation and many others; however, in this paper, transient detection is used to increase the temporal resolution of the analysis filter bank in a noise reduction solution.

In Lukin et al [9], transient is defined as variance of signal properties in time and they have used the convolution of audio critical bands with an impulse response of $h(t)=\{-1,-1,-1,0,1,1,1\}$ and the estimation of transient-like signals is achieved by thresholding of these sub-band values.

In the edge preserving method used in image noise reduction[12], the transient behavior of the noisy signal is represented with the value $S_{k}$ as defined in equation (5), this value and comparison of it with the value of $T_{k}$ controls the increase or decrease size of the frame size Where $E_{k}$ represents the energy of the noisy signal in the $k^{\text {th }}$ frame, $M_{k}$ is the average of the noisy signal in the $k^{\text {th }}$ frame, $\delta n_{k}$ is the noise variance in the $k^{\text {th }}$ frame and $L_{k}$ is the frame length. This method can be a low complexity and efficient algorithm for transient detection in audio signals.

$$
S_{k}=E_{k}-\left(M_{k}\right)^{2}-\left(\delta n_{k}\right)^{2}, \quad T_{k}=\eta \frac{\left(\delta n_{k}\right)^{2}}{L_{k}}
$$

Transient detection in speech is also used for efficient high quality compression of speech signals. In one implementation [15], the weighted summation of the first derivative of signal energy in four frequency regions of up to $5.5 \mathrm{kHz}$ is used as a measure to mark the transient segment of the speech signal. The transient is also detected by a likelihood ratio measurement of statistical similarity of two block of data to decide whether the signal has stayed stationary or its statistical characteristics have changed, hence signifying a transient event [10].

One efficient method to detect the transient of speech was suggested by Li et al [16], who have defined the transient in a time series as non-recurrence signals with lack of fractal structure, using the computational complexity for detection of nonlinear features of the signal in the time series.

In this study a benchmark between methods presented in [12], [15] and [16] was carried out. The method in [16] provided the best results, hence the implementation details of this algorithm and its modification (underlined section) from [16] is listed below: 
$>$ In a received audio buffer, compute the mean of the signal and construct a binary sequence by comparing the value of the audio buffer to the mean value.

$>$ Estimate the Kolmogorov Complexity ("KC") of the binary sequence.

$>\quad$ Calculate the first order derivative of $\mathrm{KC}$ and compare the absolute value of the first derivative with a threshold.

$>\quad$ Hold the activated flag for $32 \mathrm{~ms}$ to ensure that transient signal is included during the active flag period.

Figure1 below shows the ability of this method to detect the transient start of speech when speech is mixed with white noise, with an input SNR value of 12 .

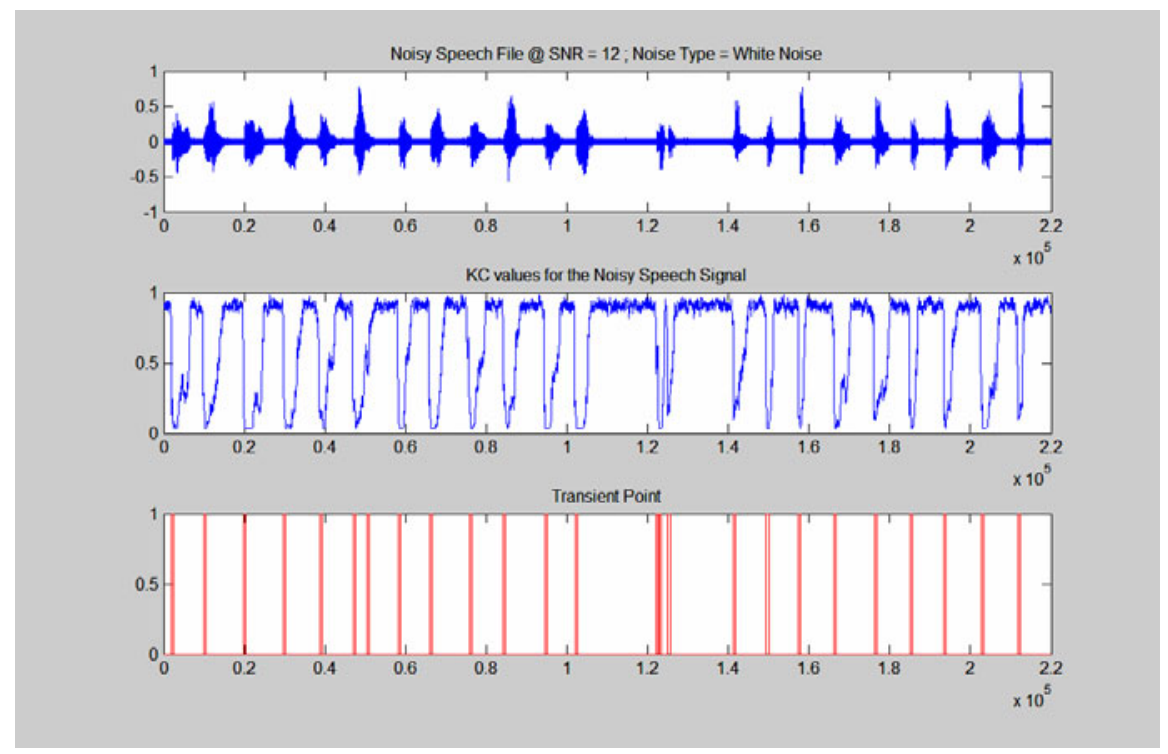

Fig. 1. Noisy Speech Signal, KC value of the signal and Transient Point in the Noisy Speech Signal

\section{Noise Reduction Modified System}

The primary objective in this paper is to preserve the transient point in the speech audio signal by increasing the temporal resolution of the analysis filter bank. The secondary objective is to illustrate the advantages of a time-domain implementation to achieve this purpose.

By using the $\mathrm{KC}$ modified method described above, we are able to detect the transient points in speech and upon detection of the transient; the analysis filter bank will be switched to provide a better temporal resolution. When the transient point ends, the analysis filter bank is switched back to an analysis filter bank with a better frequency resolution so optimum noise reduction of the algorithm can be achieved. In this implementation, as the noise reduction is carried out in time domain, there is no 
constraint to ensure the full reconstruction of the filter bank because the frequency domain signal does not need to be synthesized back to the time domain (the optimal spectral coefficients are synthesised in time domain as explained in section 2). The only consideration is given to the group delay of two filters resulting from high frequency resolution versus high temporal resolution analysis filter banks; this can be simply rectified by zero-padding the filters achieved with a high temporal resolution filter bank. Figure 2 below illustrates a sample filter achieved from the two filter banks $(\mathrm{L} 1=32 \& \mathrm{~L} 2=128)$ and their group delays.

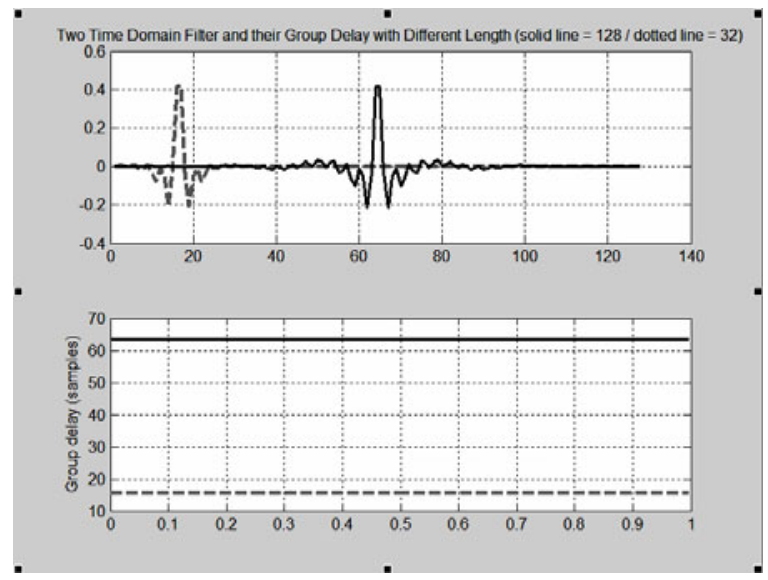

Fig. 2. Time Domain Filters and their group delays

In the above example, in order to equalise the group delay and avoid any glitches or discontinuity in the processed signal, the filter implemented with a high temporal resolution filter bank is padded with zeros, where the required number of zeros is $\frac{L 2-L 1}{2}=48$.

In figure 3, the overall structure of the proposed method is depicted. The input data is buffered and passed through one of the two analysis filter banks. The selection of the analysis filter bank depends on the $\mathrm{KC}$ transient detection block. The windowed signal is then passed through a STFT to determine the optimal spectral gains [5],[6],[13],[14]. Additionally, the transient detection flag controls the smoothing performed on the optimal spectral filter gains and signal spectra. If the transient is detected, then the spectral gains will change their values more rapidly in order to take into account the transient nature of the signal [11] and in other circumstances, the spectral gains are smoothed further with an auto regressive filter to provide a steady and consistent noise reduction performance.

Once the optimal spectral filter is determined, the filter is mapped into the time domain and is convolved with the incoming noisy audio signal. If the transient signal is detected, a variable gain of between 0 and $4 \mathrm{~dB}$ is also applied to the processed audio signal. Tests based on informal subjective listening suggest that higher gain values ( $4 \mathrm{~dB}$ ) can be applied at high SNR values, however this gain needs to be reduced as the 
SNR deteriorates. A similar method is suggested in [17] to improve the intelligibility of speech signal in the presence of background noise and deployed in [10] for a noise reduction algorithm with a switching analysis/synthesis filter bank structure.

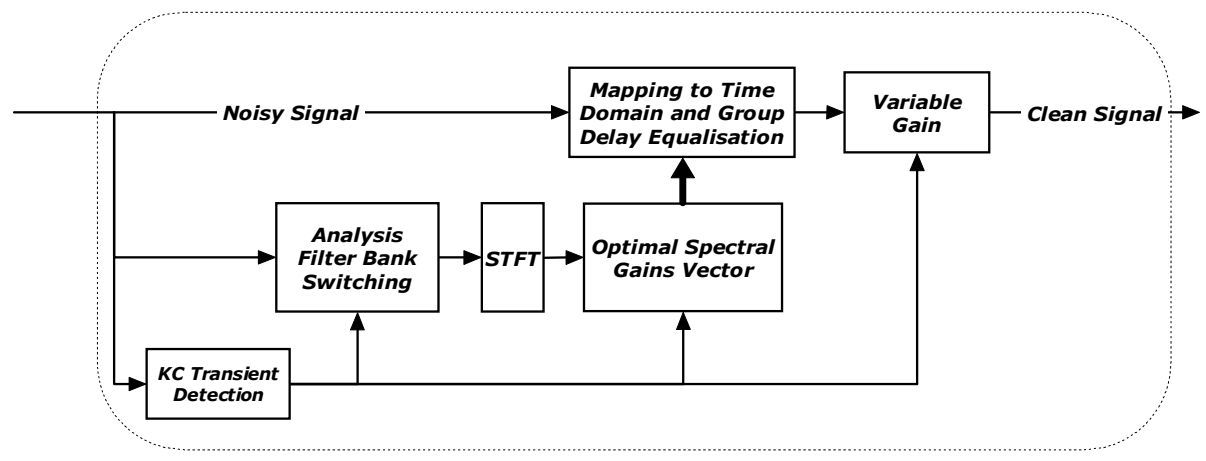

Fig. 3. Overall Structure of Suggested Method

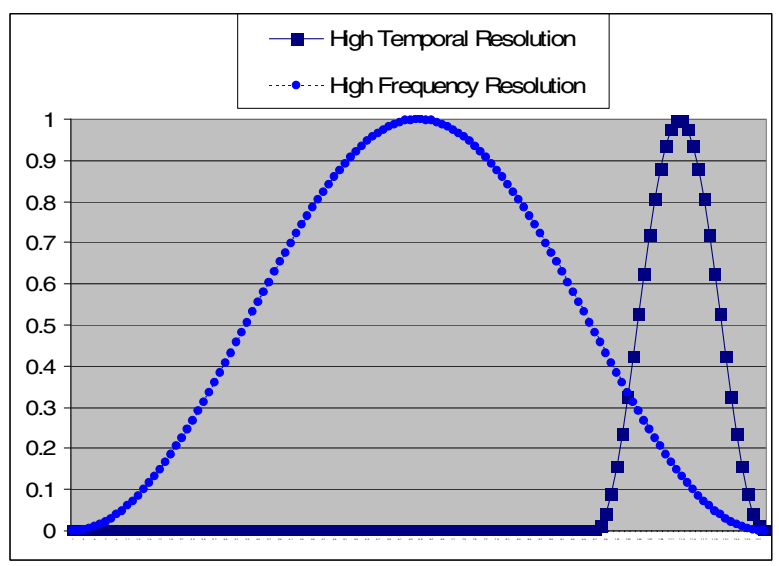

Fig. 4. Two Selections of Windows for Higher Temporal ( $\square$ ) / Frequency Resolution (

\section{Results}

Figure 5 below, illustrates a pure speech signal (top left image); noisy speech signal with pink noise @ SNR 15 (bottom left); processed signal with fixed analysis (top right); and the proposed method of switching analysis in the time domain (bottom right). As can be seen, with the proposed noise reduction method, the transient speech signals are less attenuated in comparison with a noise reduction algorithm with a fixed analysis filter bank.

The proposed algorithm was tested objectively against a fixed time/frequency resolution analysis filter bank; the test database included two male and two female speakers and 80 different words spoken by different male and female speakers. The 
noise types were selected to be white noise, pink noise, car road noise and engine noise at different SNR values of 9, 12, 15 and 20 (at lower SNR values, the performance of the transient detector algorithm deteriorates, which impacts the effectiveness of the algorithm). The objective measure for benchmarking the proposed algorithm is PESQ (Perceptual Evaluation of Speech Quality) [4]. The objective measurement (The PESQ software for objective evaluation of noise reduction algorithm can be downloaded from [18]) shows a significant improvement for the proposed method compared to noise reduction with fixed time/frequency resolution. This is illustrated in figure 6 where the PESQ value is increased by 0.1 on average for a variety of noise types and at different input SNR values.

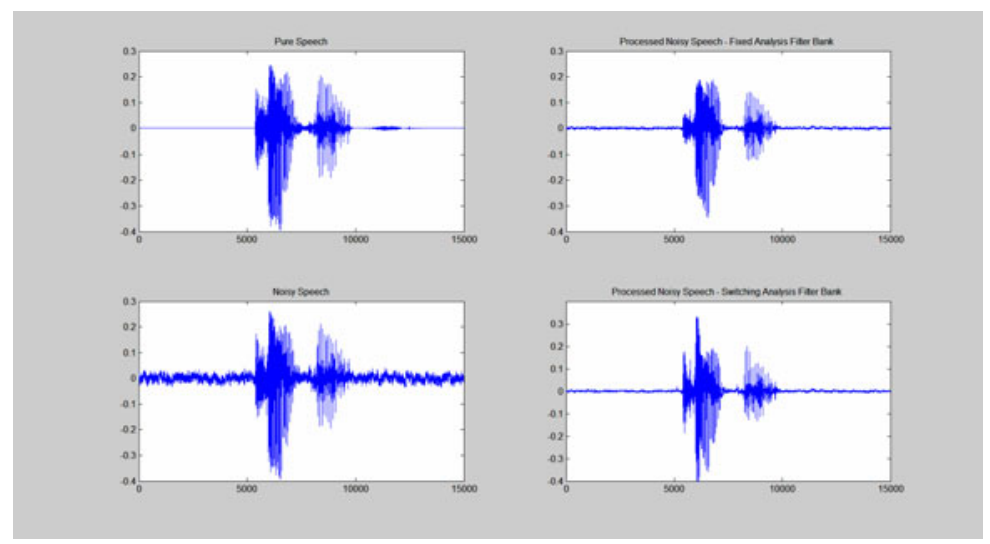

Fig. 5. Time Domain presentations of Clean, Noisy and Processed Signals

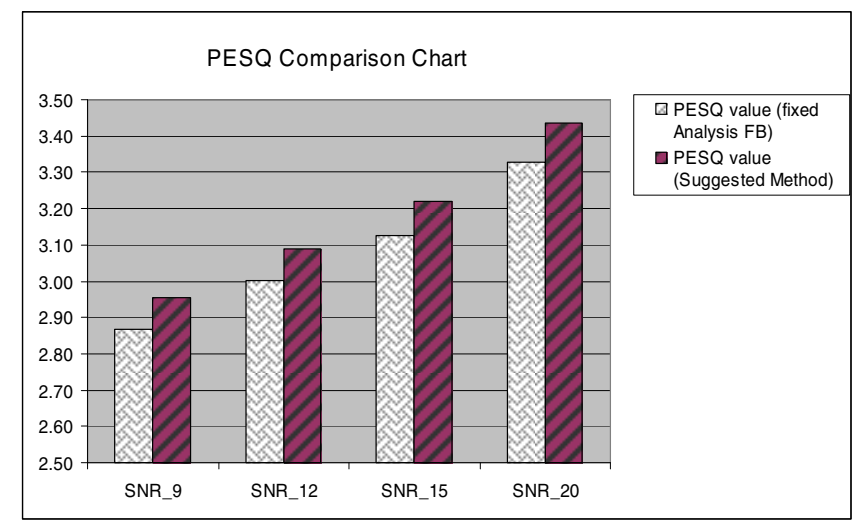

Fig. 6. PESQ values for fixed analysis filter bank and suggested methods

\section{Conclusion}

In this paper, a method to preserve the transient element of speech in a noise reduction system is proposed. $\mathrm{KC}$ values were used to identify the transient 
components of speech and upon detection of these transient events, the temporal resolution of analysis filter bank used for noise reduction was increased. Objective measurement shows superior performance of the proposed methods over a standard noise reduction method with a fixed analysis filter bank. The objective performance evaluation was based on PESQ and gave an increase of 0.1 PESQ values on average over the standard method for a variety of noisy test signals.

The proposed algorithm is performed in the time domain, which results in no need for a synthesis filter bank. This eliminates any requirement for full reconstruction of the selected filter bank during switching period and reduces the complexity, computational load and memory footprint of the algorithm. Additionally, as the noise reduction is performed in the time domain, the only processing delay is due to the group delay of the time domain filter, which is small $(<8 \mathrm{msec})$ and therefore makes the proposed algorithm a suitable solution for many applications that are sensitive to processing delay in the receive and/or transmit channels.

Acknowledgment. The authors would like to thank EPSRC for funding the research under research grant EE385538.

\section{References}

[1] Martin, R.: Noise power spectral density estimation based on optimal smoothing and minimum statistics. IEEE Trans. on Speech and Audio Processing 9(5), 504-512 (2001)

[2] Cohen, I., Berdugo, B.: Noise estimation by minima controlled recursive averaging for robust speech enhancement. IEEE Signal Proc. Letters 9(1), 12-15 (2002)

[3] Cohen, I.: Noise Spectrum Estimation in Adverse Environments: Improved Minima Controlled Recursive Averaging. IEEE Transactions On Speech And Audio Processing 11(5) (2003)

[4] Louizo, P.: Speech Enhancement Theory and Practice. CRC Press, Boca Raton (2007)

[5] Kamath, S.D., Loizou, P.C.: A Multi-Band Spectral Subtraction Method For Enhancing Speech Corrupted By Colored Noise. In: ICASSP (2002)

[6] Hu, Y., Loizou, P.: Speech enhancement by Wavelet thresholding the multitaper spectrum. IEEE Transactions on Speech and Audio Processing 12(1), 59-67 (2004)

[7] Hendriks, R.C., Heusdens, R., Jensen, J.: Adaptive time segmentation for improved speech enhancement. IEEE Transactions on Audio, Speech, and Language Processing 14(6), 2064-2074 (2006)

[8] Kauppinen, I., Roth, K.: Improved Noise Reduction in Audio Signals Using Spectral Resolution Enhancement With Time-Domain Signal Extrapolation. IEEE Transactions On Speech And Audio Processing 13(6) (2005)

[9] Lukin, A., Todd, J.: Adaptive Time-Frequency Resolution for Analysis and Processing of Audio. In: AES 120th Convention, Paris, France (2006)

[10] Mauler, D., Martin, R.: Improved Reproduction of Stops in Noise Reduction Systems with Adaptive Windows and Non-stationary Detection. EURASIP Journal on Advances in Signal Processing (2009)

[11] Quatieri, T.F., Dunn, R.B.: Speech Enhancement Based on Auditory Spectral Change. In: ICASSP, pp. 257-260 (2002)

[12] Song, W.-J., Pexrlman, W.A.: Edge-Preserving Noise Filtering Based on Adaptive Windowing. IEEE Transactions On Circuits And Systems 35(8) (1988) 
[13] Gustafsson, H.: Speech enhancement for mobile communications. Department of Telecommunications and Signal processing, University of Karlskrona/Ronneby, Ronneby (2000)

[14] Gustafsson, et al: Signal Noise Reduction By Time_Domain Spectral Subtraction. US Patent US6507623B1 (2003)

[15] Szwoch, G., Kulesza, M., Czyżewski, A.: Transient Detection for Speech Coding Applications. IJCSNS International Journal of Computer Science and Network Security 6(12) (2006)

[16] Li, Y., Fan, Y.-L., Tong, Q.-Y.: Endpoint Detection In Noisy Environment Using Complexity Measure. In: Proceedings of the 2007 International Conference on Wavelet Analysis and Pattern Recognition, Beijing, China (2007)

[17] Hazan, V., Simpson, A.: The effect of cue-enhancement on the intelligibility of nonsense word and sentence materials presented in noise. Speech Communication 24(3), 211-226 (1998)

[18] PESQ and other objective measures for evaluating quality of speech, http://www.utdallas.edu/ loizou/speech/software.htm 